\title{
On the birational invariance of the BCOV torsion of Calabi-Yau threefolds
}

\author{
V. Maillot* \& D. Rössler ${ }^{\dagger}$
}

\begin{abstract}
In [9, Sec. 4, Conj. 4.17] Fang, Lu and Yoshikawa conjecture that a certain spectral string-theoretic invariant of Calabi-Yau threefolds (the BCOV torsion) is a birational invariant. We prove a weak form of this conjecture. The proof combines the arithmetic Riemann-Roch theorem in Arakelov geometry with some inputs from motivic integration theory.
\end{abstract}

Keywords: mirror symmetry; birational geometry; Riemann-Roch; motivic integration; Calabi-Yau; Arakelov

2010 Mathematics Subject Classification(s): 14J32; 14E18; 14J33; $14 \mathrm{G} 40$

to Eckart Viehweg, in memoriam

\section{Introduction}

Let $Y$ be a smooth projective variety of dimension 3 over $\mathbb{C}$. We suppose that $Y$ is a CalabiYau variety (in the restricted sense); by definition, this means that $H^{1}\left(Y, \mathcal{O}_{Y}\right)=H^{2}\left(Y, \mathcal{O}_{Y}\right)=$ 0 and that $\omega_{Y}:=\operatorname{det}\left(\Omega_{Y}\right) \simeq \mathcal{O}_{Y}$. In view of the symmetry of Hodge numbers and Serre duality, this entails that the Hodge diamond of $Y$ is a cross.

\footnotetext{
*Institut de Mathématiques de Jussieu, Université Paris 7 Denis Diderot, C.N.R.S., Case Postale 7012, 2 place Jussieu, F-75251 Paris Cedex 05, FRANCE, E-mail : vmaillot@math.jussieu.fr

†Institut de Mathématiques, Équipe Emile Picard, Université Paul Sabatier, 118 Route de Narbonne, 31062 Toulouse cedex 9, FRANCE, E-mail: rossler@math.univ-toulouse.fr
} 
In [9] (see also [24, Sec. 2]), H. Fang, Z. Lu and K.-I. Yoshikawa introduced the analytic invariant $\tau_{\mathrm{BCOV}}(Y(\mathbb{C})) \in \mathbb{R}_{+}^{*}$. See [9, p. 177] or Definition 2.3 below for the precise definition. They conjectured the following (see [9, Sec. 4, Conj. 4.17] and [24, Sec. 2. Conj. 2.1]): if $Y$ and $Y^{\prime}$ are birational Calabi-Yau varieties of dimension 3 over $\mathbb{C}$, then

$$
\tau_{\mathrm{BCOV}}(Y(\mathbb{C}))=\tau_{\mathrm{BCOV}}\left(Y^{\prime}(\mathbb{C})\right)
$$

H. Fang, Z. Lu and K.-I. Yoshikawa explain that the quantity $\tau_{\mathrm{BCOV}}(Y(\mathbb{C}))$ is a variant of the quantity $F_{1}(Y(\mathbb{C}))$ introduced by the string-theorists M. Bershadsky, S. Cecotti, H. Ooguri and C.Vafa in [3] and [4]. The latter suggested (see [3, note added, p. 295] and [4]) that $F_{1}(Y)$ has a spectral interpretation. This was made more precise by Fang, Lu and Yoshikawa in their definition of the invariant $\tau_{\mathrm{BCOV}}(Y(\mathbb{C}))$.

The conjecture of Fang, $\mathrm{Lu}$ and Yoshikawa can be traced back to string-theory: the main invariants entering the formulation of mirror symmetry in dimension 3 should be insensitive to birationnal equivalence. For Gromov-Witten invariants of any genus this is essentially proven in [16, Theorem A and Corollary A.1, p. 155]. The mirror of Li and Ruan's statement in genus one is Fang, $\mathrm{Lu}$ and Yoshikawa's conjecture.

This conjecture should also be viewed as a "secondary" analog of the conjecture (which is now a theorem of Batyrev and Kontsevich; see [2]) that the Hodge numbers of $Y(\mathbb{C})$ and $Y^{\prime}(\mathbb{C})$ coincide.

The purpose of this note is to describe the proof of the following arithmetic result, which is a step towards the conjecture of Fang, Lu and Yoshikawa.

If $Z$ is a scheme, we shall write as usual $D^{b}(Z):=D_{c}^{b}(Z)$ for the category derived from the homotopy classes of bounded complexes of coherent sheaves on $Z$. If $\sigma: L \hookrightarrow \mathbb{C}$ is a subfield of $\mathbb{C}$, we shall write $X_{\sigma}$ for the base change $X \times_{\text {Spec } L, \sigma}$ Spec $\mathbb{C}$ of $X$ to $\mathbb{C}$ via the embedding $\sigma$.

Suppose now that $X$ (resp. $X^{\prime}$ ) is a smooth projective variety of dimension 3 over a field $L$. Suppose that $L$ is embeddable in $\mathbb{C}$ as a field and that $X$ (resp. $X^{\prime}$ ) is a Calabi-Yau variety (in the restricted sense).

Fix an embedding $\sigma: L \hookrightarrow \mathbb{C}$ and let $T$ be any finite set of embeddings $L \hookrightarrow \mathbb{C}$.

Let (S) be the statement: there exists $n \in \mathbb{N}^{*}$ and $\alpha \in L^{*}$ such that for all $\tau \in T$,

$$
\frac{\tau_{\mathrm{BCOV}}\left(X_{\tau}(\mathbb{C})\right)}{\tau_{\mathrm{BCOV}}\left(X_{\tau}^{\prime}(\mathbb{C})\right)}=\sqrt[n]{|\tau(\alpha)|} .
$$

Our main results now reads : 
Theorem 1.1. (a) If $X_{\sigma}$ is birational to $X_{\sigma}^{\prime}$ then (S) is verified.

(b) If $D^{b}\left(X_{\sigma}\right)$ and $D^{b}\left(X_{\sigma}^{\prime}\right)$ are equivalent as triangulated $\mathbb{C}$-linear categories then $(S)$ is verified.

In particular, if $L=\mathbb{Q}$ and $X_{\sigma}$ is birational to $X_{\sigma}^{\prime}$ then there exists $n \in \mathbb{N}^{*}$ such that $\left(\tau_{\mathrm{BCOV}}(X(\mathbb{C})) / \tau_{\mathrm{BCOV}}\left(X^{\prime}(\mathbb{C})\right)\right)^{n} \in \mathbb{Q}$.

Notice that a theorem of Bridgeland implies that the categories $D^{b}\left(X_{\sigma}\right)$ and $D^{b}\left(X_{\sigma}^{\prime}\right)$ are equivalent as triangulated $\mathbb{C}$-linear categories if $X_{\sigma}$ is birational to $X_{\sigma}^{\prime}$ (see [5]). Hence (b) implies (a).

We shall nevertheless give two separate proofs of (a) and (b).

Here is an outline of our proofs of (a) and (b). We first express the quantity $\tau_{\mathrm{BCOV}}$ in terms of arithmetic Chern numbers; this is made possible by the arithmetic Riemann-Roch theorem of Bismut-Gillet-Soulé [11]. To prove (a), we use Włodarczyk's theorem on the weak factorisation of birational maps (proved in [23]; see also [1]) and some lemmas describing the effect of blow-up on some global Arakelov-theoretic invariants. To prove (b), we make use of a theorem of Orlov, which asserts that if $D^{b}\left(X_{\sigma}\right)$ and $D^{b}\left(X_{\sigma}^{\prime}\right)$ are equivalent as triangulated $\mathbb{C}$-linear categories then $X_{\sigma}$ and $X_{\sigma}^{\prime}$ are related by a Fourier-Mukai functor. We also use a theorem of Caldararu, which describes the effect of a Fourier-Mukai functor on the singular cohomology of $X_{\sigma}(\mathbb{C})$ and $X_{\sigma}^{\prime}(\mathbb{C})$.

Remark. It is likely that Theorem 1.1 is true without any restriction of finiteness on $T$. In particular, the quantity $\alpha$ should not depend on $T$. The reason for restricting the statement to finite $T$ is a (probably unnecessary) hypothesis of finiteness included in the definition of an arithmetic ring in Arakelov geometry.

Acknowledgments. We thank J.-L. Colliot-Thélène, C. Voisin and K.-I. Yoshikawa for interesting mathematical discussions on matters related to this article. We are also very grateful to the referee for the close attention he paid to the text.

\section{The invariant $\tau_{\mathrm{BCOV}}$ and arithmetic Chern numbers on Calabi- Yau threefolds}

We shall apply the arithmetic Riemann-Roch theorem to certain vector bundles on $X$. In the following, we shall freely use the terminology of global Arakelov theory. For a concise summary of the necessary vocabulary, see [21, Sec. 1].

Let $f: X \rightarrow S:=$ Spec $L$ be the structure morphism. We may enlarge the set $T$ without changing the conclusion of Theorem 1.1, so we may assume that $T$ is conjugation-invariant. 
We view $L$ as an arithmetic ring, endowed with the set of embeddings $T$ into $\mathbb{C}$. We endow $X(\mathbb{C}):=\coprod_{\tau \in T} X_{\tau}(\mathbb{C})$ with a conjugation-invariant Kähler form $\nu$. We suppose that the class of $\nu$ in $H^{2}(X(\mathbb{C}), \mathbb{C})$ is the first Chern class of some ample line bundle on $X(\mathbb{C})$. Let $\bar{\Omega}:=\bar{\Omega}_{X}$ be the sheaf of differentials of $X$, endowed with the metric induced by $\nu$. We write $\bar{\omega}:=\bar{\omega}_{X}$ for $\operatorname{det}\left(\bar{\Omega}_{X}\right)$ and $\bar{\Omega}^{p}:=\bar{\Omega}_{X}^{p}$ for $\Lambda^{p}\left(\bar{\Omega}_{X}\right)$. Furthermore, we shall write $H^{q}\left(X, \bar{\Omega}^{p}\right)$ for the $L$ vector space $\mathrm{R}^{q} f_{*}\left(\Omega^{p}\right)$, endowed with the $L^{2}$-metric induced by $\nu$.

Let $\overline{\mathcal{E}}$ be the natural exact sequence of hermitian bundles

$$
0 \rightarrow f^{*} f_{*} \bar{\omega} \rightarrow \bar{\omega} \rightarrow 0 \rightarrow 0
$$

We let $\eta:=\widetilde{\operatorname{ch}}(\overline{\mathcal{E}})$ be the Bott-Chern secondary class associated to $\overline{\mathcal{E}}$, so that

$$
f^{*} f_{*} \bar{\omega}-\bar{\omega}=\eta
$$

in $\widehat{\mathrm{K}}_{0}(X)$, the arithmetic Grothendieck group of $X$. We shall write $\eta^{0}$ for the degree 0 part of $\eta$.

We apply the arithmetic Riemann-Roch theorem to $f$ and to the formal linear combination of hermitian bundles

$$
-\bar{\Omega}^{1}+2 \cdot \bar{\Omega}^{2}-3 \cdot \bar{\Omega}^{3} \text {. }
$$

See $[11,(1)$, p. 473] for this theorem. We obtain the equality

$$
\begin{aligned}
\widehat{\mathrm{c}}_{1}\left(\mathrm { R } \cdot f _ { * } \left[-\bar{\Omega}^{1}+2 \cdot \bar{\Omega}^{2}\right.\right. & \left.\left.-3 \cdot \bar{\Omega}^{3}\right]\right)-a\left(\tau\left(-\bar{\Omega}^{1}+2 \cdot \bar{\Omega}^{2}-3 \cdot \bar{\Omega}^{3}\right)\right) \\
=f_{*}\left(\widehat{\mathrm{Td}}\left(\bar{\Omega}^{\vee}\right) \widehat{\operatorname{ch}}\left(-\bar{\Omega}^{1}+2 \cdot \bar{\Omega}^{2}-3 \cdot \bar{\Omega}^{3}\right)\right)^{(1)} & \quad-a\left(\left[\int_{X} R(\Omega)^{\vee} \operatorname{Td}\left(\Omega^{\vee}\right) \operatorname{ch}\left(-\Omega^{1}+2 \cdot \Omega^{2}-3 \cdot \Omega^{3}\right)\right]^{(1)}\right)
\end{aligned}
$$

in $\widehat{\mathrm{CH}}^{1}(L)_{\mathbb{Q}}$, which is the first arithmetic Chow group of $L$, tensored with $\mathbb{Q}$.

Recall that $R(\cdot)$ is the $R$-genus of Gillet-Soulé and that $\tau(\cdot)$ is the Ray-Singer analytic torsion; see [11, Introduction].

We shall first analyse the various terms appearing in this equation. Write $\zeta_{\mathbb{Q}}(s)$ for the evaluation of the Riemann zeta function at $s \in \mathbb{C} \backslash\{1\}$.

Lemma 2.1. The equation

$$
\begin{aligned}
\widehat{\operatorname{Td}}\left(\bar{\Omega}^{\vee}\right) \widehat{\operatorname{ch}}\left(-\bar{\Omega}^{1}+2 \cdot \bar{\Omega}^{2}-3 \cdot \bar{\Omega}^{3}\right) \\
\quad=-\widehat{\mathrm{c}}^{\mathrm{top}}\left(\bar{\Omega}^{\vee}\right)\left[\zeta_{\mathbb{Q}}(0) \mathrm{rk}(\Omega)+\zeta_{\mathbb{Q}}(-1) \widehat{\mathrm{c}}_{1}(\bar{\omega})+\text { terms of degree }>1\right] \\
\quad=-\widehat{\mathrm{c}}^{\mathrm{top}}\left(\bar{\Omega}^{\vee}\right)\left[\zeta_{\mathbb{Q}}(0) \mathrm{rk}(\Omega)+\zeta_{\mathbb{Q}}(-1) \widehat{\mathrm{c}}_{1}\left(f^{*} f_{*} \bar{\omega}\right)-a\left(\zeta_{\mathbb{Q}}(-1) \eta^{0}\right)+\text { terms of degree }>1\right]
\end{aligned}
$$

holds in $\widehat{\mathrm{CH}}^{\bullet}(X)_{\mathbb{Q}}$. 
Proof. The proof is similar to the proof of [18, Lemma 3.1] so we omit it.

Using the projection formula together with Lemma 2.1, we obtain that

$$
\left[f_{*}\left(\widehat{\operatorname{Td}}\left(\bar{\Omega}^{\vee}\right) \widehat{\operatorname{ch}}\left(-\bar{\Omega}^{1}+2 \cdot \bar{\Omega}^{2}-3 \cdot \bar{\Omega}^{3}\right)\right)\right]^{(1)}=-\zeta_{\mathbb{Q}}(-1) \widehat{\mathrm{c}}_{1}\left(f_{*} \bar{\omega}\right) \int_{X} c^{\mathrm{top}}\left(\Omega^{\vee}\right)+a\left(\zeta_{\mathbb{Q}}(-1) \int_{X} c^{\mathrm{top}}\left(\bar{\Omega}^{\vee}\right) \eta^{0}\right)
$$

in $\widehat{\mathrm{CH}}^{1}(L)_{\mathbb{Q}}$ (here the superscript ${ }^{(1)}$ refers to part of degree 1 in $\left.\widehat{\mathrm{CH}}^{1}(L)_{\mathbb{Q}}\right)$. To understand this computation, recall that $f_{*}\left(x^{(l)}\right)=f_{*}(x)^{(l-3)}$ for all $x \in \mathrm{CH}^{\bullet}(X)_{\mathbb{Q}}$.

We have the identity of cohomology classes

$R(\Omega)^{\vee} \operatorname{Td}\left(\Omega^{\vee}\right) \operatorname{ch}\left(-\Omega^{1}+2 \cdot \Omega^{2}-3 \cdot \Omega^{3}\right)=-R\left(\Omega^{\vee}\right) c^{\text {top }}\left(\Omega^{\vee}\right)\left[\zeta_{\mathbb{Q}}(0) \operatorname{rk}(\Omega)+\right.$ terms of degree $\left.>0\right]$

and so

$$
\left[\int_{X} R(\Omega)^{\vee} \operatorname{Td}\left(\Omega^{\vee}\right) \operatorname{ch}\left(-\Omega^{1}+2 \cdot \Omega^{2}-3 \cdot \Omega^{3}\right)\right]^{(1)}=0
$$

since $R\left(\Omega^{\vee}\right)^{(1)}=0$ by assumption.

As to the left-hand side of equation (1), we have

$$
\begin{aligned}
\mathrm{R}^{\bullet} f_{*} & {\left[-\bar{\Omega}^{1}+2 \cdot \bar{\Omega}^{2}-3 \cdot \bar{\Omega}^{3}\right] } \\
= & -\left[H^{0}\left(X, \bar{\Omega}^{1}\right)-H^{1}\left(X, \bar{\Omega}^{1}\right)+H^{2}\left(X, \bar{\Omega}^{1}\right)-H^{3}\left(X, \bar{\Omega}^{1}\right)\right] \\
+ & 2\left[H^{0}\left(X, \bar{\Omega}^{2}\right)-H^{1}\left(X, \bar{\Omega}^{2}\right)+H^{2}\left(X, \bar{\Omega}^{2}\right)-H^{3}\left(X, \bar{\Omega}^{2}\right)\right] \\
& \quad-3\left[H^{0}\left(X, \bar{\Omega}^{3}\right)-H^{1}\left(X, \bar{\Omega}^{3}\right)+H^{2}\left(X, \bar{\Omega}^{3}\right)-H^{3}\left(X, \bar{\Omega}^{3}\right)\right] \\
= & -\left[-H^{1}\left(X, \bar{\Omega}^{1}\right)+H^{2}\left(X, \bar{\Omega}^{1}\right)\right]+2\left[-H^{1}\left(X, \bar{\Omega}^{2}\right)+H^{2}\left(X, \bar{\Omega}^{2}\right)\right]-3\left[H^{0}\left(X, \bar{\Omega}^{3}\right)-H^{3}\left(X, \bar{\Omega}^{3}\right)\right] .
\end{aligned}
$$

These equalities follow from the fact that the Hodge diamond of a Calabi-Yau threefold is a cross. Applying the first arithmetic Chern class to the last expression, we get

$$
\begin{aligned}
\widehat{\mathrm{c}}_{1}\left(\mathrm{R}^{\bullet}\right. & \left.f_{*}\left[-\bar{\Omega}^{1}+2 \cdot \bar{\Omega}^{2}-3 \cdot \bar{\Omega}^{3}\right]\right) \\
& =\widehat{\mathrm{c}}_{1}\left(H^{1}\left(X, \bar{\Omega}^{1}\right)-H^{2}\left(X, \bar{\Omega}^{1}\right)-2 \cdot H^{1}\left(X, \bar{\Omega}^{2}\right)+2 \cdot H^{2}\left(X, \bar{\Omega}^{2}\right)-3 \cdot H^{0}\left(X, \bar{\Omega}^{3}\right)+3 \cdot H^{3}\left(X, \bar{\Omega}^{3}\right)\right) \\
& =\widehat{\mathrm{c}}_{1}\left(-H^{1}\left(X, \bar{\Omega}^{1}\right)-H^{1}\left(X, \bar{\Omega}^{2}\right)-3 \cdot H^{0}\left(X, \bar{\Omega}^{3}\right)-3 \cdot H^{0}(X, \overline{\mathcal{O}})\right)
\end{aligned}
$$

We used the fact that Serre duality is compatible with $L^{2}$-metrics (see [12, p. 27, after eq. 9]) in the last equality.

Putting everything together, we get

$$
\begin{aligned}
& -\widehat{\mathrm{c}}_{1}\left(H^{1}\left(X, \bar{\Omega}^{1}\right)\right)-\widehat{\mathrm{c}}_{1}\left(H^{1}\left(X, \bar{\Omega}^{2}\right)\right)-3 \widehat{\mathrm{c}}_{1}\left(H^{0}(X, \overline{\mathcal{O}})\right)-3 \widehat{\mathrm{c}}_{1}\left(H^{0}\left(X, \bar{\Omega}^{3}\right)\right)-a\left(\tau \left(-\bar{\Omega}^{1}+2 \cdot \bar{\Omega}^{2}-3 \cdot \bar{\Omega}\right.\right. \\
= & -\zeta_{\mathbb{Q}}(-1) \widehat{\mathrm{c}}_{1}\left(f_{*} \bar{\omega}\right) \int_{X} c^{\mathrm{top}}\left(\Omega^{\vee}\right)+a\left(\zeta_{\mathbb{Q}}(-1) \int_{X} c^{\mathrm{top}}\left(\bar{\Omega}^{\vee}\right) \eta^{0}\right)
\end{aligned}
$$


Now notice that by definition,

$$
\widehat{\mathrm{c}}_{1}\left(H^{0}(X, \overline{\mathcal{O}})\right)=-a(\log \operatorname{Vol}(X(\mathbb{C}), \nu))
$$

where

$$
\operatorname{Vol}(X(\mathbb{C}, \nu)):=\frac{1}{3 !(2 \pi)^{3}} \int_{X(\mathbb{C})} \nu^{3} .
$$

Notice also that $f_{*} \bar{\omega}=H^{0}\left(X, \bar{\Omega}^{3}\right)$ (again by definition). Taking into account the fact that $\zeta_{\mathbb{Q}}(-1)=-1 / 12$, we see that the equality (2) can be rewritten as

$$
\begin{aligned}
-\widehat{\mathrm{c}}_{1}\left(H^{1}\left(X, \bar{\Omega}^{2}\right)\right)-\left(\frac{1}{12} \chi(X)+3\right) \widehat{\mathrm{c}}_{1}\left(H^{0}\left(X, \bar{\Omega}^{3}\right)\right) & =a\left(-\frac{1}{12} \int_{X} c^{\mathrm{top}}\left(\bar{\Omega}^{\vee}\right) \eta^{0}-3 \log \operatorname{Vol}(X(\mathbb{C}), \nu)\right) \\
& +\widehat{\mathrm{c}}_{1}\left(H^{1}\left(X, \bar{\Omega}^{1}\right)\right) \\
& +a\left(\tau\left(-\bar{\Omega}^{1}+2 \cdot \bar{\Omega}^{2}-3 \cdot \bar{\Omega}^{3}\right)\right)
\end{aligned}
$$

in $\widehat{\mathrm{CH}}^{1}(L)$. Here $\chi(X):=\int_{X} c^{\mathrm{top}}\left(\Omega^{\vee}\right)$. Note that $\chi(X)=\sum_{p, q}(-1)^{p+q} \operatorname{dim}_{L}\left(H^{q}\left(X, \Omega^{p}\right)\right)$ by the generalized Gauss-Bonnet theorem.

The $L^{2}$-metric on $H^{2}(X(\mathbb{C}), \mathbb{C})$ is induced from a Riemannian metric on the space $H^{2}(X(\mathbb{C}), \mathbb{R})$. This is a consequence of the formula [18, before Lemma 2.7]. Let $\operatorname{Vol}_{L^{2}}\left(H^{2}(X(\mathbb{C}), \mathbb{Z})\right)$ be the volume of a fundamental domain of the lattice $H^{2}(X(\mathbb{C}), \mathbb{Z})_{\text {free }}$ in $H^{2}(X(\mathbb{C}), \mathbb{R})$ for that metric. Here $H^{2}(X(\mathbb{C}), \mathbb{Z})_{\text {free }}$ is the largest direct summand of $H^{2}(X(\mathbb{C}), \mathbb{Z})$ which is a free $\mathbb{Z}$-module.

Lemma 2.2. The equality

$$
\widehat{\mathrm{c}}_{1}\left(H^{1}\left(X, \bar{\Omega}^{1}\right)\right)=a\left(-\bigoplus_{\tau \in T} \log \left(\operatorname{Vol}_{L^{2}}\left(H^{2}\left(X_{\tau}(\mathbb{C}), \mathbb{Z}\right)\right)\right)\right)
$$

holds in $\widehat{\mathrm{CH}}^{1}(L)_{\mathbb{Q}}$.

Proof. Let $\tau \in T$ and let $e_{1}, \ldots, e_{r}$ be a basis of $H^{2}\left(X_{\tau}(\mathbb{C}), \mathbb{Z}\right)_{\text {free. }}$ By definition, we have

$$
\operatorname{Vol}_{L^{2}}\left(H^{2}\left(X_{\tau}(\mathbb{C}), \mathbb{Z}\right)\right)=\left|e_{1} \wedge \cdots \wedge e_{r}\right|^{2}
$$

where $|\cdot|$ refers to the natural norm on $\Lambda^{r}\left(H^{2}(X(\mathbb{C}), \mathbb{C})\right)$. Since $H^{2}\left(X_{\tau}(\mathbb{C}), \mathbb{C}\right) \simeq H^{1,1}(X(\mathbb{C}))$ by hypothesis, we may conclude from the Lefschetz theorem on $(1,1)$-classes that the elements $e_{i}$ are classes of algebraic cycles $\mathbf{e}_{\mathbf{i}}$ on $X_{\tau}$. Let $\underline{\tau}_{0}: K \hookrightarrow \mathbb{C}$ be a field of definition for the $\mathbf{e}_{\mathbf{i}}$, where $\underline{\tau}_{0}$ extends $\tau$. We may assume that $K$ is finite over $L$ (see [8, proof of Prop. 1.5]). Write $\mathbf{e}_{\mathbf{i}}{ }^{K}$ for the model of $\mathbf{e}_{\mathbf{i}}$ in $X_{K}$ and write $\mathrm{cl}_{\mathrm{dR}}$ for the cycle class map with values 
in de Rham cohomology. Let $\underline{\tau}: K \hookrightarrow \mathbb{C}$ be another embedding of $K$ extending $\tau$. Since by construction

$$
H^{2}\left(X_{K, \underline{\tau}}(\mathbb{C}), \mathbb{C}\right) \simeq H_{\mathrm{dR}}^{2}\left(X_{K} / K\right) \otimes_{\underline{\tau}} \mathbb{C}
$$

we see that the elements $\mathrm{cl}_{\mathrm{dR}}\left(\mathbf{e}_{\mathbf{i}}^{K}\right) \otimes_{\underline{\tau}} 1$ form a basis of $H^{2}\left(X_{K, \underline{\tau}}(\mathbb{C}), \mathbb{C}\right)$. Furthermore, since $\mathrm{cl}_{\mathrm{dR}}\left(\mathbf{e}_{\mathbf{i}}{ }^{K}\right) \otimes_{\underline{\tau}} 1=\mathrm{cl}_{\mathrm{dR}}\left(\mathbf{e}_{\mathbf{i}}{ }^{K} \otimes_{\underline{\tau}} \mathbb{C}\right)$, we see that the elements $\mathrm{cl}_{\mathrm{dR}}\left(\mathbf{e}_{\mathbf{i}}{ }^{K}\right) \otimes_{\underline{\tau}} 1$ even form a basis of $H^{2}\left(X_{K, \underline{\tau}}(\mathbb{C}), \mathbb{Z}\right)_{\text {free. }}$ Furthermore, there is a natural identification

$$
H^{2}\left(X_{K, \underline{\tau}}(\mathbb{C}), \mathbb{Z}\right)_{\text {free }} \simeq H^{2}\left(X_{\tau}(\mathbb{C}), \mathbb{Z}\right)_{\text {free }}
$$

which is an isometry for the $L^{2}$-metrics.

Now let $f:$ Spec $K \rightarrow$ Spec $L$ be the natural map. We view Spec $K$ as an arithmetic variety over Spec $L$. By the above, we have the equalities

$$
\begin{aligned}
\widehat{\mathrm{c}}_{1}\left(H^{1}\left(X_{K}, \bar{\Omega}^{1}\right)\right) & =a\left(-\bigoplus_{\underline{\tau} \mid \tau, \tau \in T} \log \left|\operatorname{cl}\left(\mathbf{e}_{\mathbf{1}}{ }^{K}\right) \otimes_{\underline{\underline{\tau}}} 1 \wedge \cdots \wedge \mathrm{cl}\left(\mathbf{e}_{\mathbf{r}}{ }^{K}\right) \otimes_{\underline{\tau}} 1\right|^{2}\right) \\
& =a\left(-\bigoplus_{\underline{\tau} \mid \tau, \tau \in T} \log \operatorname{Vol}_{L^{2}}\left(X_{\tau}(\mathbb{C}), \mathbb{Z}\right)\right)
\end{aligned}
$$

and thus

$$
\begin{aligned}
{[K: L] \widehat{\mathrm{c}}_{1}\left(H^{1}\left(X, \bar{\Omega}^{1}\right)\right) } & =f_{*} f^{*} \widehat{\mathrm{c}}_{1}\left(H^{1}\left(X, \bar{\Omega}^{1}\right)\right)=f_{*} \widehat{\mathrm{c}}_{1}\left(H^{1}\left(X_{K}, \bar{\Omega}^{1}\right)\right) \\
& =[K: L] a\left(-\bigoplus_{\tau \in T} \log \operatorname{Vol}_{L^{2}}\left(X_{\tau}(\mathbb{C}), \mathbb{Z}\right)\right)
\end{aligned}
$$

and we can conclude.

The previous calculations motivate the following definition :

Definition 2.3 (BCOV torsion).

$$
\begin{aligned}
\tau_{\mathrm{BCOV}}(X(\mathbb{C})):=\exp [ & -\frac{1}{12} \int_{X(\mathbb{C})} c^{\mathrm{top}}\left(\bar{\Omega}_{X(\mathbb{C})}^{\vee}\right) \eta^{0}-3 \log \operatorname{Vol}(X(\mathbb{C}), \nu) \\
& \left.-\log \left(\operatorname{Vol}_{L^{2}}\left(H^{2}(X(\mathbb{C}), \mathbb{Z})\right)\right)-\tau\left(\bar{\Omega}_{X(\mathbb{C})}^{1}\right)+2 \tau\left(\bar{\Omega}_{X(\mathbb{C})}^{2}\right)-3 \tau\left(\bar{\Omega}_{X(\mathbb{C})}^{3}\right)\right]
\end{aligned}
$$

It is proven in $\left[9\right.$, Sec. 4.4] that $\tau_{\mathrm{BCOV}}(X)$ does not depend on the choice of $\nu$. The following equation summarizes the calculations made in this section:

$$
a\left(\log \left(\tau_{\mathrm{BCOV}}(X(\mathbb{C}))\right)\right)=-\widehat{\mathrm{c}}_{1}\left(H^{1}\left(X, \bar{\Omega}^{2}\right)\right)-\left(\frac{1}{12} \chi(X)+3\right) \widehat{\mathrm{c}}_{1}\left(H^{0}\left(X, \bar{\Omega}^{3}\right)\right) \text { in } \widehat{\mathrm{CH}}^{1}(L)_{\mathbb{Q}}
$$




\section{Proof of Theorem 1.1}

With the equation (4) in hand, we see that the conclusion of Theorem 1.1 is equivalent to the equation

$$
-\widehat{\mathrm{c}}_{1}\left(H^{1}\left(X, \bar{\Omega}^{2}\right)\right)-\left(\frac{1}{12} \chi(X)+3\right) \widehat{\mathrm{c}}_{1}\left(H^{0}\left(X, \bar{\Omega}^{3}\right)\right)=-\widehat{\mathrm{c}}_{1}\left(H^{1}\left(X^{\prime}, \bar{\Omega}^{2}\right)\right)-\left(\frac{1}{12} \chi\left(X^{\prime}\right)+3\right) \widehat{\mathrm{c}}_{1}\left(H^{0}\left(X^{\prime}, \bar{\Omega}^{3}\right)\right)
$$

Lemma 3.1. Let $L^{\prime}$ be a finite field extension of $L$. We view Spec $L^{\prime}$ as an arithmetic variety over $L$. With this convention, the assertion that the equation

$-\widehat{\mathrm{c}}_{1}\left(H^{1}\left(X_{L^{\prime}}, \bar{\Omega}^{2}\right)\right)-\left(\frac{1}{12} \chi\left(X_{L^{\prime}}\right)+3\right) \widehat{\mathrm{c}}_{1}\left(H^{0}\left(X_{L^{\prime}}, \bar{\Omega}^{3}\right)\right)=-\widehat{\mathrm{c}}_{1}\left(H^{1}\left(X_{L^{\prime}}^{\prime}, \bar{\Omega}^{2}\right)\right)-\left(\frac{1}{12} \chi\left(X_{L^{\prime}}^{\prime}\right)+3\right) \widehat{\mathrm{c}}_{1}\left(H^{0}\left(X_{L^{\prime}}^{\prime}, \bar{\Omega}^{3}\right)\right.$

is satisfied in $\widehat{\mathrm{CH}}^{1}\left(L^{\prime}\right)_{\mathbb{Q}}$ is equivalent to the assertion that the equation (5) is satisfied in $\widehat{\mathrm{CH}}^{1}(L)_{\mathbb{Q}}$.

Proof. Let $f:$ Spec $L^{\prime} \rightarrow$ Spec $L$ be the natural morphism. Using the projection formula, we compute

$$
\left[L^{\prime}: L\right] \widehat{\mathrm{c}}_{1}\left(H^{1}\left(X, \bar{\Omega}^{2}\right)\right)=f_{*} f^{*} \widehat{\mathrm{c}}_{1}\left(H^{1}\left(X, \bar{\Omega}^{2}\right)\right)=f_{*} \widehat{\mathrm{c}}_{1}\left(H^{1}\left(X_{L^{\prime}}, \bar{\Omega}^{2}\right)\right)
$$

and similarly

$$
\left[L^{\prime}: L\right] \widehat{\mathrm{c}}_{1}\left(H^{0}\left(X, \bar{\Omega}^{3}\right)\right)=f_{*} f^{*} \widehat{\mathrm{c}}_{1}\left(H^{0}\left(X, \bar{\Omega}^{3}\right)\right)=f_{*} \widehat{\mathrm{c}}_{1}\left(H^{0}\left(X_{L^{\prime}}, \bar{\Omega}^{3}\right)\right)
$$

If we combine these formulae with the analogous formulae for $X^{\prime}$, we may conclude.

Now notice that the group $\widehat{\mathrm{CH}}^{1}\left(L^{\prime}\right)_{\mathbb{Q}}$ (where $L^{\prime}$ is viewed as an arithmetic variety over $L$ ) is naturally isomorphic to the homonymous group $\widehat{\mathrm{CH}}^{1}\left(L^{\prime}\right)_{\mathbb{Q}}:=\widehat{\mathrm{CH}}^{1}\left(L^{\prime}, T^{\prime}\right)_{\mathbb{Q}}$, which is the first arithmetic Grothendieck group of the arithmetic ring $L^{\prime}$, endowed with the set

$$
T^{\prime}:=\left\{\tau^{\prime}: L^{\prime} \hookrightarrow \mathbb{C}\left|\tau^{\prime}\right|_{L} \in T\right\}
$$

of embeddings into $\mathbb{C}$. Furthermore, this isomorphism is compatible with the formation of arithmetic Chern classes (the proof of this fact is left to the reader). Thus Lemma 3.1 implies that the truth value of Theorem 1.1 remains unchanged if we replace $L$ by a finite extension field $L^{\prime}$ and $T$ by the set $T^{\prime}:=\left\{\tau^{\prime}: L^{\prime} \hookrightarrow \mathbb{C}\left|\tau^{\prime}\right|_{L} \in T\right\}$.

Before we begin with the proof, notice that by the formula [18, before Lemma 2.7], the $L^{2}$ metric on $H^{1}\left(X, \bar{\Omega}^{2}\right)$ is given by the formula

$$
\langle\lambda, \kappa\rangle_{L^{2}}=\frac{i}{(2 \pi)^{3}} \int_{X(\mathbb{C})} \lambda \wedge \bar{\kappa}
$$


and the $L^{2}$-metric on $H^{0}\left(X, \bar{\Omega}^{3}\right)$ is given by the formula

$$
\langle\lambda, \kappa\rangle_{L^{2}}=\frac{-i}{(2 \pi)^{3}} \int_{X(\mathbb{C})} \lambda \wedge \bar{\kappa}
$$

In particular, these metrics do not depend on the choice of the Kähler form $\nu$.

\subsection{Proof of Theorem 1.1 (a)}

We now assume that there is a birational transformation from $X_{\sigma}$ to $X_{\sigma}^{\prime}$. Recall that $\sigma: L \rightarrow \mathbb{C}$ is a fixed embedding of $L$ into $\mathbb{C}$ (see before Theorem 1.1).

Lemma 3.2. There is a birational transformation from $X_{\bar{L}}$ to $X_{\bar{L}}^{\prime}$.

Proof. This can be proven using a "spreading out" argument. We leave the details to the reader.

Notice that the birational transformation provided by the last Lemma has a model over a finite extension of $L$. Hence, by the discussion following Lemma 3.1, we may assume without loss of generality that there is a birational transformation from $X$ to $X^{\prime}$ defined over $L$.

Lemma 3.3.

$$
\widehat{\mathrm{c}}_{1}\left(H^{0}\left(X, \bar{\Omega}^{3}\right)\right)=\widehat{\mathrm{c}}_{1}\left(H^{0}\left(X^{\prime}, \bar{\Omega}^{3}\right)\right)
$$

Proof. Let $\phi$ be a birational transformation from $X$ to $X^{\prime}$. It is shown in [14, Proof of Th. 8.19, chap. II] that there is an open set $U \subseteq X$ and a morphism $f: U \rightarrow X^{\prime}$, with the following properties : $f$ induces $\phi$ and codimension $(U) \leqslant 2$. It is also shown in [14, Proof of Th. 8.19, chap. II] that the maps

$$
H^{0}\left(X^{\prime}, \Omega^{3}\right) \stackrel{f^{*}}{\longrightarrow} H^{0}\left(U, \Omega^{3}\right) \stackrel{\text { restriction to } U}{\longleftarrow} H^{0}\left(X, \Omega^{3}\right)
$$

are bijective. Thus, using the formula (7), we compute that

$$
\widehat{\mathrm{c}}_{1}\left(H^{0}\left(X^{\prime}, \bar{\Omega}^{3}\right)\right)=-a\left(\log \left|\int_{X^{\prime}(\mathbb{C})} \lambda \wedge \bar{\lambda}\right|\right)=-a\left(\log \left|\int_{X(\mathbb{C})} f^{*}(\lambda) \wedge \overline{f^{*}(\lambda)}\right|\right)=\widehat{\mathrm{c}}_{1}\left(H^{0}\left(X, \bar{\Omega}^{3}\right)\right)
$$

Here $\lambda \in H^{0}\left(X^{\prime}, \Omega^{3}\right)$ is any non-zero element.

We recall the following theorem of Manin (and others). 
Theorem 3.4. Let $Y$ be a smooth projective variety over $\mathbb{C}$. Let $Z \hookrightarrow Y$ be a smooth closed subvariety of codimension $c$ of $Y$. Let $\psi: \widetilde{Y}:=\mathrm{Bl}_{Z}(Y) \rightarrow Y$ be the blow-up of $Y$ along $Z$. Let $e: E \hookrightarrow \widetilde{Y}$ be the immersion of the exceptional divisor and let $\pi: E \rightarrow Z$ be the natural morphism. Let $\mathcal{O}_{E}(1)$ be the tautological vector bundle on $E$. For any $k \in \mathbb{N}$, there is a natural isomorphism of $\mathbb{Q}$-vector spaces

$$
H^{k}(Y(\mathbb{C}), \mathbb{Q}) \oplus \bigoplus_{l \geqslant 0}^{c-2} H^{k-2 l}(Z(\mathbb{C}), \mathbb{Q})(-l-1) \stackrel{\sim}{\rightarrow} H^{k}(\widetilde{Y}(\mathbb{C}), \mathbb{Q})
$$

which respects the corresponding Hodge structures. This isomorphism is given by the formula

$$
\begin{aligned}
&\left(\eta, \kappa_{0}, \ldots, \kappa_{c-2}\right) \\
& \longmapsto\left(\psi^{*} \eta, e_{*}\left[\pi^{*}\left(\kappa_{0}\right)+\pi^{*}\left(\kappa_{1}\right) \cdot c_{1}\left(\mathcal{O}_{E}(1)\right)+\pi^{*}\left(\kappa_{2}\right) \cdot c_{1}\left(\mathcal{O}_{E}(1)\right)^{2}+\cdots\right.\right. \\
&\left.\left.\cdots+\pi^{*}\left(\kappa_{c-2}\right) \cdot c_{1}\left(\mathcal{O}_{E}(1)\right)^{c-2}\right]\right) .
\end{aligned}
$$

Proof. See [19].

Lemma 3.5. Let $C$ be a non-singular curve of genus $g$ over $L$. Then

$$
\widehat{\mathrm{c}}_{1}\left(H^{0}\left(\operatorname{Jac}(C), \bar{\Omega}^{g}\right)\right)=\widehat{\mathrm{c}}_{1}\left(H^{0}\left(C, \bar{\Omega}^{1}\right)\right)+(g-1) \log (2 \pi)
$$

in $\widehat{\mathrm{CH}}^{1}(L)$, for any Kähler metrics on $C(\mathbb{C})$ and $\operatorname{Jac}(C)(\mathbb{C})$.

Proof. See [22, Exp. I, Lemme 3.2.1].

Proposition 3.6. Let $Y$ be a smooth projective threefold over L. Let $Z \hookrightarrow Y$ be a smooth closed subcurve of genus $g$ of $Y$. Let $\phi: \widetilde{Y}:=\mathrm{Bl}_{Z}(Y) \rightarrow Y$ be the blow-up of $Y$ along $Z$. Then

$$
\widehat{\mathrm{c}}_{1}\left(H^{1}\left(\widetilde{Y}, \bar{\Omega}^{2}\right)\right)=\widehat{\mathrm{c}}_{1}\left(H^{1}\left(Y, \bar{\Omega}^{2}\right)\right)+\widehat{\mathrm{c}}_{1}\left(H^{0}\left(Z, \bar{\Omega}^{1}\right)\right)+2 g \log (2 \pi)
$$

for any Kähler metrics on $Y, Z$ and $\tilde{Y}$.

Proof. Let $e: E \hookrightarrow \widetilde{Y}$ be the immersion of the exceptional divisor. Let $\pi: E \rightarrow Z$ be the natural morphism. By Theorem 3.4, the map

$$
H^{1}\left(Y, \Omega^{2}\right) \oplus H^{0}(Z, \Omega) \longrightarrow H^{1}\left(\widetilde{Y}, \Omega^{2}\right)
$$

given by the formula

$$
(\eta, \kappa) \longmapsto \phi^{*}(\eta)+e_{*}\left(\pi^{*}(\kappa)\right)
$$


is an isomorphism. We compute

$$
\begin{aligned}
& \frac{i}{(2 \pi)^{3}} \int_{\widetilde{Y}(\mathbb{C})}\left(\phi^{*}\left(\eta_{1}\right)+e_{*}\left(\pi^{*}\left(\kappa_{1}\right)\right) \wedge\left(\phi^{*}\left(\overline{\eta_{2}}\right)+e_{*}\left(\pi^{*}\left(\overline{\kappa_{2}}\right)\right)\right)\right. \\
= & \frac{i}{(2 \pi)^{3}} \int_{\widetilde{Y}(\mathbb{C})} \phi^{*}\left(\eta_{1}\right) \wedge \phi^{*}\left(\overline{\eta_{2}}\right)+\frac{i}{(2 \pi)^{3}} \int_{\widetilde{Y}(\mathbb{C})} e_{*} \pi^{*}\left(z^{*}\left(\eta_{1}\right) \wedge \bar{\kappa}_{2}\right) \\
& \quad+\frac{i}{(2 \pi)^{3}} \int_{\widetilde{Y}(\mathbb{C})} e_{*} \pi^{*}\left(z^{*}\left(\bar{\eta}_{2}\right) \wedge \kappa_{1}\right)+\frac{i}{(2 \pi)^{3}} \int_{\widetilde{Y}(\mathbb{C})} e_{*}\left(\pi^{*}\left(\kappa_{1}\right)\right) \wedge e_{*}\left(\pi^{*}\left(\bar{\kappa}_{2}\right)\right) \\
= & \frac{i}{(2 \pi)^{3}} \int_{\widetilde{Y}(\mathbb{C})} \phi^{*}\left(\eta_{1}\right) \wedge \phi^{*}\left(\overline{\eta_{2}}\right)+\frac{i}{(2 \pi)^{3}} \int_{\widetilde{Y}(\mathbb{C})} e_{*}\left(\pi^{*}\left(\kappa_{1}\right)\right) \wedge e_{*}\left(\pi^{*}\left(\bar{\kappa}_{2}\right)\right) .
\end{aligned}
$$

Now using the self-intersection formula (see for instance [10, VI, 1., 1.4.2]), we may compute

$$
\begin{aligned}
& \frac{i}{(2 \pi)^{3}} \int_{\widetilde{Y}(\mathbb{C})} e_{*}\left(\pi^{*}\left(\kappa_{1}\right)\right) \wedge e_{*}\left(\pi^{*}\left(\bar{\kappa}_{2}\right)\right)=\frac{i}{(2 \pi)^{3}} \int_{E(\mathbb{C})} e_{*}\left(\pi^{*}\left(\kappa_{1}\right) \wedge e^{*} e_{*}\left(\pi^{*}\left(\bar{\kappa}_{2}\right)\right)\right) \\
= & \frac{i}{(2 \pi)^{3}} \int_{E(\mathbb{C})} e_{*}\left(c_{1}\left(\mathcal{O}_{E}(-1)\right) \wedge \pi^{*}\left(\kappa_{1}\right) \wedge \pi^{*}\left(\bar{\kappa}_{2}\right)\right) \\
= & -\frac{i}{(2 \pi)^{3}} \int_{Z(\mathbb{C})} \kappa_{1} \wedge \bar{\kappa}_{2}=\frac{1}{(2 \pi)^{2}} \cdot \frac{-i}{2 \pi} \int_{Z(\mathbb{C})} \kappa_{1} \wedge \bar{\kappa}_{2} .
\end{aligned}
$$

These formulae imply the conclusion of the proposition.

Lemma 3.7. Let $A$ and $B$ be abelian varieties over $\bar{L}$ and let $\phi: A_{\sigma} \rightarrow B_{\sigma}$ be an isogeny (over $\left.\mathbb{C}\right)$ ). Then there is an isogeny $A \rightarrow B$ (over $\bar{L})$.

Proof. By spreading out. Left to the reader.

Lemma 3.8. Let $A$ and $B$ be two abelian varieties over $L$ and suppose that there exists an isogeny $\phi: A \rightarrow B$ (over $L$ ). Suppose that $L$ contains a square root of $\operatorname{deg}(\phi)$. Then

$$
\widehat{\mathrm{c}}_{1}\left(H^{0}\left(A, \bar{\Omega}^{g}\right)\right)=\widehat{\mathrm{c}}_{1}\left(H^{0}\left(B, \bar{\Omega}^{g}\right)\right)
$$

in $\widehat{\mathrm{CH}}^{1}(L)$, for any choice of Kähler metrics on $A(\mathbb{C})$ and $B(\mathbb{C})$.

Proof. Let $\alpha_{1}, \ldots, \alpha_{g}$ be a basis of the $L$-vector space $H^{0}(B, \Omega)$. Using the formula [18, before Lemma 2.7], we see that for any embedding $\tau \in T$, we have

$$
\begin{aligned}
\left\langle\alpha_{1} \wedge \alpha_{2} \wedge \cdots\right. & \left.\wedge \alpha_{g}, \alpha_{1} \wedge \alpha_{2} \wedge \cdots \wedge \alpha_{g}\right\rangle_{L^{2}} \\
& =\int_{B(\mathbb{C})}\left(\left(\alpha_{1} \wedge \alpha_{2} \wedge \cdots \wedge \alpha_{g}\right) \otimes_{\tau} 1\right) \wedge \overline{\left(\left(\alpha_{1} \wedge \alpha_{2} \wedge \cdots \wedge \alpha_{g}\right) \otimes_{\tau} 1\right)} \\
& =\operatorname{deg}(\phi)^{-1} \int_{A(\mathbb{C})}\left(\left(\phi^{*}\left(\alpha_{1}\right) \wedge \phi^{*}\left(\alpha_{2}\right) \wedge \cdots \wedge \phi^{*}\left(\alpha_{g}\right)\right) \otimes_{\tau} 1\right) \\
& \wedge \overline{\left(\left(\phi^{*}\left(\alpha_{1}\right) \wedge \phi^{*}\left(\alpha_{2}\right) \wedge \cdots \wedge \phi^{*}\left(\alpha_{g}\right)\right) \otimes_{\tau} 1\right)}
\end{aligned}
$$


and thus the mapping $H^{0}\left(B, \Omega^{g}\right) \rightarrow H^{0}\left(A, \Omega^{g}\right)$ given by the formula $\eta \mapsto(\sqrt{\operatorname{deg}(\phi)}) \cdot \phi^{*}$ is an isometry of hermitian vector bundles.

Let now $\phi: X \rightarrow X^{\prime}$ be a birational transformation. Let $X^{\prime \prime}$ be another smooth projective variety over $L$, together with morphisms $f: X^{\prime \prime} \rightarrow X$ and $g: X^{\prime \prime} \rightarrow X^{\prime}$ such that $\phi \circ f$ and $g$ coincide as birational transformations. The variety $X^{\prime \prime}$ can be obtained as a desingularisation of the Zariski closure of the graph of $\phi$ in $X \times X^{\prime}$.

Denote by $\mathcal{P} H S(\mathbb{Q})$ the category of (pure) polarisable $\mathbb{Q}$-Hodge structures.

Using weak factorisation of birational maps (see [1]), Theorem 3.4 and Proposition 3.6 we conclude that after possibly replacing $L$ by one of its finite extensions, there are curves $C_{1}, \ldots, C_{r^{\prime}}$ defined over $L$ and numbers $s_{l}^{\prime} \in\{0,1\}$ so that

$$
H^{3}\left(X_{\sigma}(\mathbb{C}), \mathbb{Q}\right)+\sum_{l=1}^{r^{\prime}}(-1)^{s_{l}^{\prime}} H^{1}\left(C_{l, \sigma}^{\prime}, \mathbb{Q}\right)(-1)=H^{3}\left(X_{\sigma}^{\prime \prime}(\mathbb{C}), \mathbb{Q}\right)
$$

in $K_{0}(\mathcal{P} H S(\mathbb{Q}))$ and so that

$$
\widehat{\mathrm{c}}_{1}\left(H^{1}\left(X, \bar{\Omega}^{2}\right)\right)+\sum_{l=1}^{r^{\prime}}(-1)^{s_{l}^{\prime}} \widehat{\mathrm{c}}_{1}\left(H^{0}\left(C_{l}^{\prime}, \bar{\Omega}^{1}\right)\right)+2 \sum_{l=1}^{r^{\prime}}(-1)^{s_{l}^{\prime}} \operatorname{genus}\left(C_{l}^{\prime}\right) \log (2 \pi)=\widehat{\mathrm{c}}_{1}\left(H^{1}\left(X^{\prime \prime}, \bar{\Omega}^{2}\right)\right) .
$$

Symmetrically, there are curves $C_{1}^{\prime \prime}, \ldots, C_{r^{\prime \prime}}^{\prime \prime}$ over $L$ and numbers $s_{l}^{\prime \prime} \in\{0,1\}$ so that

$$
H^{3}\left(X_{\sigma}^{\prime}(\mathbb{C}), \mathbb{Q}\right)+\sum_{l=1}^{r^{\prime \prime}}(-1)^{s_{l}^{\prime \prime}} H^{1}\left(C_{l, \sigma}^{\prime \prime}, \mathbb{Q}\right)(-1)=H^{3}\left(X_{\sigma}^{\prime \prime}(\mathbb{C}), \mathbb{Q}\right)
$$

in $K_{0}(\mathcal{P} H S(\mathbb{Q}))$ and so that

$\widehat{\mathrm{c}}_{1}\left(H^{1}\left(X^{\prime}, \bar{\Omega}^{2}\right)\right)+\sum_{l=1}^{r^{\prime \prime}}(-1)^{s_{l}^{\prime \prime}} \widehat{\mathrm{c}}_{1}\left(H^{0}\left(C_{l}^{\prime \prime}, \bar{\Omega}^{1}\right)\right)+2 \sum_{l=1}^{r^{\prime \prime}}(-1)^{s_{l}^{\prime \prime}} \operatorname{genus}\left(C_{l}^{\prime \prime}\right) \log (2 \pi)=\widehat{\mathrm{c}}_{1}\left(H^{1}\left(X^{\prime \prime}, \bar{\Omega}^{2}\right)\right)$.

Now by a theorem of Kontsevich (proved using motivic integration; see [17]) there is an isomorphism of $\mathbb{Q}$-Hodge structures $H^{3}\left(X_{\sigma}(\mathbb{C}), \mathbb{Q}\right) \simeq H^{3}\left(X_{\sigma}^{\prime}(\mathbb{C}), \mathbb{Q}\right)$. Thus

$$
2 \sum_{l=1}^{r^{\prime}}(-1)^{s_{l}^{\prime}} \text { genus }\left(C_{l}^{\prime}\right) \log (2 \pi)=2 \sum_{l=1}^{r^{\prime \prime}}(-1)^{s_{l}^{\prime \prime}} \text { genus }\left(C_{l}^{\prime \prime}\right) \log (2 \pi) \text {. }
$$

Furthermore, since the category of polarisable $\mathbb{Q}$-Hodge structures is semi-simple (see for instance [7, Lemme 4.2.3]), there exists an isomorphism of $\mathbb{Q}$-Hodge structures

$$
\bigoplus_{l: s_{l}^{\prime}=1} H^{1}\left(\operatorname{Jac}\left(C_{l}^{\prime}\right)_{\sigma}, \mathbb{Q}\right) \oplus \bigoplus_{l: s_{l}^{\prime \prime}=0} H^{1}\left(\operatorname{Jac}\left(C_{l}^{\prime \prime}\right)_{\sigma}, \mathbb{Q}\right) \rightarrow \bigoplus_{l: s_{l}^{\prime}=0} H^{1}\left(\operatorname{Jac}\left(C_{l}^{\prime}\right)_{\sigma}, \mathbb{Q}\right) \oplus \bigoplus_{l: s_{l}^{\prime \prime}=1} H^{1}\left(\operatorname{Jac}\left(C_{l}^{\prime \prime}\right)_{\sigma}, \mathbb{Q}\right)
$$


and thus an $\bar{L}$-isogeny $\gamma$ of abelian varieties

$$
\prod_{l: s_{l}^{\prime}=1} \operatorname{Jac}\left(C_{l}^{\prime}\right) \prod \prod_{l: s_{l}^{\prime \prime}=0} \operatorname{Jac}\left(C_{l}^{\prime \prime}\right) \rightarrow \prod_{l: s_{l}^{\prime}=0} \operatorname{Jac}\left(C_{l}^{\prime}\right) \prod \prod_{l: s_{l}^{\prime \prime}=1} \operatorname{Jac}\left(C_{l}^{\prime \prime}\right)
$$

Here we used Lemma 3.7. Extend $L$ further so that $\gamma$ is defined over $L$ and so that $L$ contains a square root of $\operatorname{deg}(\gamma)$. Then, by Lemma 3.8, we have

$$
\sum_{l}^{r^{\prime}}(-1)^{s_{l}^{\prime}} \widehat{\mathrm{c}}_{1}\left(H^{0}\left(\operatorname{Jac}\left(C_{l}^{\prime}\right), \bar{\Omega}^{\operatorname{genus}\left(C_{l}^{\prime}\right)}\right)=\sum_{l}^{r^{\prime \prime}}(-1)^{s_{l}^{\prime \prime}} \widehat{\mathrm{c}}_{1}\left(H^{0}\left(\operatorname{Jac}\left(C_{l}^{\prime \prime}\right), \bar{\Omega}^{\operatorname{genus}\left(C_{l}^{\prime \prime}\right)}\right) .\right.\right.
$$

in $\widehat{\mathrm{CH}}^{1}(L)_{\mathbb{Q}}$. Using Lemma 3.5, we deduce that

$$
\sum_{l}^{r^{\prime}}(-1)^{s_{l}^{\prime}} \widehat{\mathrm{c}}_{1}\left(H^{0}\left(C_{l}^{\prime}, \bar{\Omega}^{1}\right)\right)=\sum_{l}^{r^{\prime \prime}}(-1)^{s_{l}^{\prime \prime}} \widehat{\mathrm{c}}_{1}\left(H^{0}\left(C_{l}^{\prime \prime}, \bar{\Omega}^{1}\right)\right)
$$

so that

$$
\widehat{\mathrm{c}}_{1}\left(H^{1}\left(X, \bar{\Omega}^{2}\right)\right)=\widehat{\mathrm{c}}_{1}\left(H^{1}\left(X^{\prime}, \bar{\Omega}^{2}\right)\right) .
$$

Furthermore, by Lemma 3.3, we have

$$
\widehat{\mathrm{c}}_{1}\left(H^{0}\left(X, \bar{\Omega}^{3}\right)\right)=\widehat{\mathrm{c}}_{1}\left(H^{0}\left(X^{\prime}, \bar{\Omega}^{3}\right)\right)
$$

and again by a theorem of Kontsevich (see [17]) we have $\chi(X)=\chi\left(X^{\prime}\right)$. This implies that $-\widehat{\mathrm{c}}_{1}\left(H^{1}\left(X, \bar{\Omega}^{2}\right)\right)-\left(\frac{1}{12} \chi(X)+3\right) \widehat{\mathrm{c}}_{1}\left(H^{0}\left(X, \bar{\Omega}^{3}\right)\right)=-\widehat{\mathrm{c}}_{1}\left(H^{1}\left(X^{\prime}, \bar{\Omega}^{2}\right)\right)-\left(\frac{1}{12} \chi\left(X^{\prime}\right)+3\right) \widehat{\mathrm{c}}_{1}\left(H^{0}\left(X^{\prime}, \bar{\Omega}^{3}\right)\right)$ in $\widehat{\mathrm{CH}}^{1}(L)_{\mathbb{Q}}$. Thus the equation (5) is verified and the theorem is proved.

\subsection{Proof of Theorem 1.1 (b)}

We now assume that the categories $D^{b}\left(X_{\sigma}\right)$ and $D^{b}\left(X_{\sigma}^{\prime}\right)$ are equivalent as triangulated $\mathbb{C}$ linear categories.

As a matter of notation, if $X_{1} \times X_{2} \times \cdots \times X_{t}$ is a cartesian product of varieties and $1 \leqslant i_{1}<i_{2}<\cdots<i_{j} \leqslant t$, we shall write

$$
\pi_{X_{i_{1}} X_{i_{2}} \cdots X_{i_{j}}}^{X_{1} X_{2} \ldots X_{t}}: X_{1} \times X_{2} \times \cdots \times X_{t} \longrightarrow X_{i_{1}} \times X_{i_{2}} \times \cdots \times X_{i_{j}}
$$

for the natural projection.

We shall make use of the following theorems. 
Theorem 3.9 (Orlov). There exists an object $M$ (resp. $\left.M^{\prime}\right)$ in $D^{b}\left(X_{\sigma} \times X_{\sigma}^{\prime}\right)$ with the following properties.

(a) The object

$$
\mathrm{R}^{\bullet} \pi_{X_{\sigma} X_{\sigma}, *}^{X_{\sigma} X_{\sigma}^{\prime} X_{\sigma}}\left(\pi_{X_{\sigma} X_{\sigma}^{\prime}}^{X_{\sigma} X_{\sigma}^{\prime} X_{\sigma}, *}(M) \underline{\otimes} \pi_{X_{\sigma}^{\prime} X_{\sigma}}^{X_{\sigma} X_{\sigma}^{\prime} X_{\sigma}, *}\left(M^{\prime}\right)\right)
$$

is isomorphic in $D^{b}\left(X_{\sigma} \times X_{\sigma}\right)$ to the structure sheaf of the diagonal in $X_{\sigma} \times X_{\sigma}$.

(b) The object

$$
\mathrm{R}^{\bullet} \pi_{X_{\sigma}^{\prime} X_{\sigma}^{\prime}, *}^{X^{\prime} X_{\sigma} X_{\sigma}^{\prime}}\left(\pi_{X_{\sigma}^{\prime} X_{\sigma}}^{X^{\prime} X_{\sigma} X_{\sigma}^{\prime}, *}\left(M^{\prime}\right) \underline{\otimes} \pi_{X_{\sigma} X_{\sigma}^{\prime}}^{X_{\sigma}^{\prime} X_{X_{\sigma}}^{\prime}, *}(M)\right)
$$

is isomorphic in $D^{b}\left(X_{\sigma}^{\prime} \times X_{\sigma}^{\prime}\right)$ to the structure sheaf of the diagonal in $X_{\sigma}^{\prime} \times X_{\sigma}^{\prime}$.

Proof. See [20].

The last theorem is actually valid more generally if $X_{\sigma}$ (resp. $X_{\sigma}^{\prime}$ ) is replaced by any smooth quasi-projective scheme over $\mathbb{C}$ and if one assumes that $D^{b}\left(X_{\sigma}\right)$ and $D^{b}\left(X_{\sigma}^{\prime}\right)$ are equivalent as triangulated $\mathbb{C}$-linear categories.

Write $\pi: X_{\sigma} \times X_{\sigma}^{\prime} \rightarrow X_{\sigma}$ for the first projection and $\pi^{\prime}: X_{\sigma} \times X_{\sigma}^{\prime} \rightarrow X_{\sigma}^{\prime}$ for the second projection.

Theorem 3.10 (Căldăraru). Let $M$ and $M^{\prime}$ be objects satisfying the conditions $(a)$ and $(b)$ in Theorem 3.9, then the map

$$
\Phi_{M}^{H}: H^{\bullet}\left(X_{\sigma}(\mathbb{C}), \mathbb{Q}\right) \longrightarrow H^{\bullet}\left(X_{\sigma}^{\prime}(\mathbb{C}), \mathbb{C}\right)
$$

given by the formula

$$
\left.\Phi_{M}^{H}(\beta):=\pi_{*}^{\prime}\left(\pi^{*}(\beta) \cdot \sqrt{\mathrm{Td}}\left(X_{\sigma} \times X_{\sigma}^{\prime}\right) \cdot \operatorname{ch}(M)\right)\right)
$$

is an isomorphism and for any $k \in \mathbb{N}$ we have

$$
\Phi_{M}^{H}\left(\oplus_{p-q=k} H^{p, q}\left(X_{\sigma}(\mathbb{C})\right)\right)=\oplus_{p-q=k} H^{p, q}\left(X_{\sigma}^{\prime}(\mathbb{C})\right) .
$$

Proof. See [6] or [15, par. 5.2].

Theorem 3.10 is actually valid more generally if $X_{\sigma}$ (resp. $X_{\sigma}^{\prime}$ ) is replaced by any smooth projective scheme over $\mathbb{C}$.

Corollary 3.11. Let the notations and hypotheses of Theorem 3.10 hold. Then we have

$$
\Phi_{M}^{H}\left(H^{p, q}\left(X_{\sigma}(\mathbb{C})\right)\right)=H^{p, q}\left(X_{\sigma}^{\prime}(\mathbb{C})\right) .
$$

for any $p, q \in \mathbb{N}$ such that $p+q=3$. 
Proof. Using the fact that $X_{\sigma}(\mathbb{C})$ is a Calabi-Yau variety, we see that there are identifications

$$
\oplus_{p-q=3} H^{p, q}\left(X_{\sigma}(\mathbb{C})\right)=H^{3,0}\left(X_{\sigma}(\mathbb{C})\right), \quad \oplus_{p-q=1} H^{p, q}\left(X_{\sigma}(\mathbb{C})\right)=H^{2,1}\left(X_{\sigma}(\mathbb{C})\right)
$$

and

$$
\oplus_{p-q=-1} H^{p, q}\left(X_{\sigma}(\mathbb{C})\right)=H^{1,2}\left(X_{\sigma}(\mathbb{C})\right), \quad \oplus_{p-q=-3} H^{p, q}\left(X_{\sigma}(\mathbb{C})\right)=H^{0,3}\left(X_{\sigma}(\mathbb{C})\right) .
$$

Similar identities hold for $X_{\sigma}^{\prime}(\mathbb{C})$ in place of $X_{\sigma}(\mathbb{C})$. This implies the conclusion of the Corollary.

Theorem 3.12 (Căldăraru). Let the notations and hypotheses of Theorem 3.10 hold. Then we have

$$
\int_{X(\mathbb{C})} \beta \wedge \lambda=\int_{X^{\prime}(\mathbb{C})} \Phi_{M}^{H}(\beta) \wedge \Phi_{M}^{H}(\lambda)
$$

for all $\beta, \lambda \in H^{3}\left(X_{\sigma}(\mathbb{C}), \mathbb{C}\right)$.

Proof. In the presence of Corollary 3.11, this is a special case of the formula given in [15, Prop. 5.44, p. 133].

Lemma 3.13. There exists a finite field extension $K$ of $L$ and an object $M_{0}\left(\right.$ resp. $\left.M_{0}^{\prime}\right)$ of $D^{b}\left(X_{K} \times X_{K}^{\prime}\right)$ (resp. $D^{b}\left(X_{K}^{\prime} \times X_{K}\right)$ ) such that

(a) ${ }_{K}$ The object

$$
\mathrm{R} \cdot \pi_{X_{K} X_{K}, *}^{X_{K} X_{K}^{\prime} X_{K}}\left(\pi_{X_{K} X_{K}^{\prime}}^{X_{K} X_{K}^{\prime} X_{K}, *}(M) \underline{\otimes} \pi_{X_{K}^{\prime} X_{K}}^{X_{K} X_{K}^{\prime} X_{K}, *}\left(M^{\prime}\right)\right)
$$

is isomorphic in $D^{b}\left(X_{K} \times X_{K}\right)$ to the structure sheaf of the diagonal in $X_{K} \times X_{K}$.

(b) $K$ The object

$$
\mathrm{R} \bullet \pi_{X_{K}^{\prime} X_{K}^{\prime}, *}^{X_{K}^{\prime} X_{K} X_{K}^{\prime}}\left(\pi_{X_{K}^{\prime} X_{K}}^{X_{X}^{\prime} X_{K} X_{K}^{\prime}, *}\left(M^{\prime}\right) \underline{\otimes} \pi_{X_{K} X_{K}^{\prime}}^{X_{K}^{\prime} X_{K} X_{K}^{\prime}, *}(M)\right)
$$

is isomorphic in $D^{b}\left(X_{K}^{\prime} \times X_{K}^{\prime}\right)$ to the structure sheaf of the diagonal in $X_{K}^{\prime} \times X_{K}^{\prime}$.

Proof. Let $\Delta: X_{\sigma} \hookrightarrow X_{\sigma} \times X_{\sigma}$ ( resp. $\Delta^{\prime}: X_{\sigma}^{\prime} \hookrightarrow X_{\sigma}^{\prime} \times X_{\sigma}^{\prime}$ ) be the diagonal morphism. Let $U$ be a bounded complex of locally free sheaves on $X_{\sigma} \times X_{\sigma}^{\prime}$ representing $M$ and let $U^{\prime}$ be a bounded complex of locally sheaves on $X_{\sigma}^{\prime} \times X_{\sigma}$ representing $M^{\prime}$. Let $L_{1}$ be a finitely generated extension of $L$ (as a field), such that $U$ (resp. $U^{\prime}$ ) has a model over $X_{L_{1}} \times_{L_{1}} X_{L_{1}}^{\prime}$ (resp. $X_{L_{1}}^{\prime} \times_{L_{1}} X_{L_{1}}$ ). Let $S$ be an affine variety over $L$, which is smooth and irreducible and whose function field is isomorphic to $L_{1}$ as an $L$-algebra. After possibly replacing $S$ by one of its open affine subsets, we may find bounded complexes of locally free sheaves $\widetilde{U}$ (resp. $\widetilde{U}^{\prime}$ ) on $X_{S} \times{ }_{S} X_{S}^{\prime}$ (resp. $\left.X_{S}^{\prime} \times{ }_{S} X_{S}\right)$, which are models of $U$ and $U^{\prime}$.

The conditions (a) and (b) in Theorem 3.9 are equivalent to the conditions : 
- There are isomorphisms of coherent sheaves

$$
\mathrm{R}^{0} \pi_{X_{\sigma} X_{\sigma}, *}^{X_{\sigma} X_{\sigma}^{\prime} X_{\sigma}}\left(\pi_{X_{\sigma} X_{\sigma}^{\prime}}^{X_{\sigma} X_{\sigma}^{\prime} X_{\sigma}, *}(M) \underline{\otimes} \pi_{X_{\sigma}^{\prime} X_{\sigma}}^{X_{\sigma} X_{\sigma}^{\prime} X_{\sigma}, *}\left(M^{\prime}\right)\right) \simeq \Delta_{*}\left(\mathcal{O}_{X_{\sigma}}\right)
$$

and

$$
\mathrm{R}^{i} \pi_{X_{\sigma} X_{\sigma}, *}^{X_{\sigma} X_{\sigma}^{\prime} X_{\sigma}}\left(\pi_{X_{\sigma} X_{\sigma}^{\prime}}^{X_{\sigma} X_{\sigma}^{\prime} X_{\sigma}, *}(M) \underline{\otimes} \pi_{X_{\sigma}^{\prime} X_{\sigma}}^{X_{\sigma} X_{\sigma}^{\prime} X_{\sigma}, *}\left(M^{\prime}\right)\right) \simeq 0
$$

for all $i \neq 0$;

- there are isomorphisms of coherent sheaves

$$
\mathrm{R}^{0} \pi_{X_{\sigma}^{\prime} X_{\sigma}^{\prime}, *}^{X_{\sigma}^{\prime} X_{\sigma} X_{\sigma}^{\prime}}\left(\pi_{X_{\sigma}^{\prime} X_{\sigma}}^{X_{\sigma}^{\prime} X_{\sigma} X_{\sigma}^{\prime}, *}\left(M^{\prime}\right) \underline{\otimes} \pi_{X_{\sigma} X_{\sigma}^{\prime}}^{X_{\sigma}^{\prime} X_{\sigma} X_{\sigma}^{\prime}, *}(M)\right) \simeq \Delta_{*}\left(\mathcal{O}_{X_{\sigma}^{\prime}}\right)
$$

and

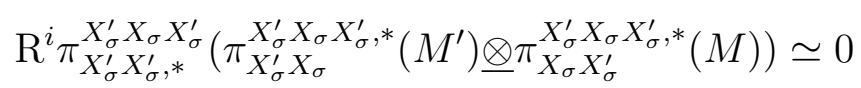

for all $i \neq 0$.

Thus, after possibly a further reduction of the size of $S$, we may assume that

- there are isomorphisms of coherent sheaves

$$
\mathrm{R}^{0} \pi_{X_{S} X_{S}, *}^{X_{S} X_{S}^{\prime} X_{S}}\left(\pi_{X_{S} X_{S}^{\prime}}^{X_{S} X_{S}^{\prime} X^{*}}(\widetilde{U}) \otimes \pi_{X_{S}^{\prime} X_{S}}^{X_{S} X_{S}^{\prime} X_{S}, *}\left(\widetilde{U}^{\prime}\right)\right) \simeq \Delta_{*} \mathcal{O}_{X_{S}}
$$

and

$$
\mathrm{R}^{i} \pi_{X_{S} X_{S}, *}^{X_{S} X_{S}^{\prime} X_{S}}\left(\pi_{X_{S} X_{S}^{\prime}}^{X_{S} X_{S}^{\prime} X_{S}, *}(\widetilde{U}) \otimes \pi_{X_{S}^{\prime} X_{S}}^{X_{S} X_{S}^{\prime} X_{S}, *}\left(\widetilde{U^{\prime}}\right)\right) \simeq 0
$$

for all $i \neq 0$;

- there are isomorphisms of coherent sheaves

$$
\mathrm{R}^{0} \pi_{X_{S}^{\prime} X_{S}^{\prime}, *}^{X_{S}^{\prime} X_{S} X_{S}^{\prime}}\left(\pi_{X_{S}^{\prime} X_{S}}^{X_{S}^{\prime} X_{S} X_{S}^{\prime}, *}\left(\widetilde{U}^{\prime}\right) \otimes \pi_{X_{S} X_{S}^{\prime}}^{X_{S}^{\prime} X_{S} X_{S}^{\prime}, *}(\widetilde{U})\right) \simeq \Delta_{*} \mathcal{O}_{X_{S}^{\prime}}
$$

and

$$
\mathrm{R}^{i} \pi_{X_{S}^{\prime} X_{S}^{\prime}, *}^{X_{S}^{\prime} X_{S} X_{S}^{\prime}}\left(\pi_{X_{S}^{\prime} X_{S}}^{X_{S}^{\prime} X_{S} X_{S}^{\prime}, *}\left(\widetilde{U}^{\prime}\right) \otimes \pi_{X_{S} X_{S}^{\prime}}^{X_{S}^{\prime} X_{S} X_{S}^{\prime}, *}(\widetilde{U})\right) \simeq 0
$$

for all $i \neq 0$.

To see this, use the fact that the elements of the complexes $\widetilde{U}$ and $\widetilde{U}^{\prime}$ are locally free and apply the theorem on cohomology and base-change (see [13, chap. III, 7.7.4]).

Now pick a closed point $s \in S$. The field $K:=\kappa(s)$ has all the properties we are looking for.

Now replace $L$ by a finite extension $K$ satisfying the conclusion of Lemma 3.13. Replace $T$ by the set $T_{K}$ of embeddings of $K$ into $\mathbb{C}$ lying above embeddings in $T$. Recall that by Lemma 3.1, this does not restrict generality. 
Proposition 3.14. There are isometries of hermitian vector bundles

$$
H^{1}\left(X, \bar{\Omega}^{2}\right) \simeq H^{1}\left(X^{\prime}, \bar{\Omega}^{2}\right)
$$

and

$$
H^{0}\left(X, \bar{\Omega}^{3}\right) \simeq H^{0}\left(X^{\prime}, \bar{\Omega}^{3}\right)
$$

over the arithmetic variety Spec $L$.

Proof. Let $M_{0}, M_{0}^{\prime}$ be as provided by Lemma 3.13. Set $M_{\sigma}:=M_{0} \otimes_{\sigma} \mathbb{C}$ and $M_{\sigma}^{\prime}:=M_{0}^{\prime} \otimes_{\sigma} \mathbb{C}$. Since $\mathbb{C}$ is flat as an $L$-algebra via $\sigma$, we see that $M_{\sigma}$ and $M_{\sigma}^{\prime}$ satisfy properties (a) and (b) in Theorem 3.9.

Furthermore, there are comparison isomorphisms

$$
H^{3}\left(X_{\sigma}(\mathbb{C}), \mathbb{C}\right) \simeq\left(\bigoplus_{p+q=3} H^{q}\left(X, \Omega^{p}\right)\right) \otimes_{\sigma} \mathbb{C}
$$

and

$$
H^{3}\left(X_{\sigma}^{\prime}(\mathbb{C}), \mathbb{C}\right) \simeq\left(\bigoplus_{p+q=3} H^{q}\left(X, \Omega^{p}\right)\right) \otimes_{\sigma} \mathbb{C}
$$

compatible with pull-backs, push-forwards and formation of Chern classes. We may thus conclude from Theorem 3.12 that the morphism $\bigoplus_{p+q=3} H^{q}\left(X, \Omega^{p}\right) \rightarrow \bigoplus_{p+q=3} H^{q}\left(X, \Omega^{p}\right)$ given by the formula in Hodge cohomology

$$
\Phi_{M}^{H_{\mathrm{Hdg}}}(\beta):=\pi_{*}^{\prime}\left(\pi^{*}(\beta) \cdot \sqrt{\mathrm{Td}}\left(X \times X^{\prime}\right) \cdot \operatorname{ch}\left(M_{0}\right)\right)
$$

is an isomorphism. Now by Theorem 3.12 and Corollary 3.11, for any $\tau \in T$ the maps

$$
\left.\Phi_{M}^{H_{\mathrm{Hdg}}} \otimes_{\tau} \mathbb{C}\right|_{H^{1}\left(X_{\tau}, \Omega^{2}\right)}: H^{1}\left(X_{\tau}, \Omega^{2}\right) \rightarrow H^{1}\left(X_{\tau}^{\prime}, \Omega^{2}\right)
$$

and

$$
\left.\Phi_{M}^{H_{\mathrm{Hdg}}} \otimes_{\tau} \mathbb{C}\right|_{H^{0}\left(X_{\tau}, \Omega^{3}\right)}: H^{0}\left(X_{\tau}, \Omega^{3}\right) \rightarrow H^{0}\left(X_{\tau}^{\prime}, \Omega^{3}\right)
$$

are isometries for the $L^{2}$-metrics. This implies the result.

We can now conclude the proof Theorem 1.1. Indeed, by Proposition 3.14, we have

$$
\widehat{\mathrm{c}}_{1}\left(H^{1}\left(X, \bar{\Omega}^{2}\right)\right)=\widehat{\mathrm{c}}_{1}\left(H^{1}\left(X^{\prime}, \bar{\Omega}^{2}\right)\right)
$$

and

$$
\widehat{\mathrm{c}}_{1}\left(H^{0}\left(X, \bar{\Omega}^{3}\right)\right)=\widehat{\mathrm{c}}_{1}\left(H^{0}\left(X^{\prime}, \bar{\Omega}^{3}\right)\right)
$$

in $\widehat{\mathrm{CH}}^{1}(L)_{\mathbb{Q}}$. We conclude using equation (4). 


\section{References}

[1] Dan Abramovich, Kalle Karu, Kenji Matsuki, and Jarosław Włodarczyk, Torification and factorization of birational maps, J. Amer. Math. Soc. 15 (2002), no. 3, 531-572 (electronic).

[2] Victor V. Batyrev, Birational Calabi-Yau n-folds have equal Betti numbers, New trends in algebraic geometry (Warwick, 1996), London Math. Soc. Lecture Note Ser., vol. 264, Cambridge Univ. Press, Cambridge, 1999, pp. 1-11.

[3] M. Bershadsky, S. Cecotti, H. Ooguri, and C. Vafa, Holomorphic anomalies in topological field theories, Mirror symmetry, II, AMS/IP Stud. Adv. Math., vol. 1, Amer. Math. Soc., Providence, RI, 1997, pp. 655-682.

[4] Kodaira-Spencer theory of gravity and exact results for quantum string amplitudes, Comm. Math. Phys. 165 (1994), no. 2, 311-427.

[5] Tom Bridgeland, Flops and derived categories, Invent. Math. 147 (2002), no. 3, 613-632.

[6] Andrei Căldăraru, The Mukai pairing. II. The Hochschild-Kostant-Rosenberg isomorphism, Adv. Math. 194 (2005), no. 1, 34-66.

[7] Pierre Deligne, Théorie de Hodge. II, Inst. Hautes Études Sci. Publ. Math. 40 (1971), 5-57.

[8] Pierre Deligne, James S. Milne, Arthur Ogus, and Kuang-yen Shih, Hodge cycles, motives, and Shimura varieties, Lecture Notes in Mathematics, vol. 900, Springer-Verlag, Berlin, 1982.

[9] Hao Fang, Zhiqin Lu, and Ken-Ichi Yoshikawa, Analytic torsion for Calabi-Yau threefolds, J. Differential Geom. 80 (2008), no. 2, 175-259.

[10] William Fulton and Serge Lang, Riemann-Roch algebra, Grundlehren der Mathematischen Wissenschaften [Fundamental Principles of Mathematical Sciences], vol. 277, Springer-Verlag, New York, 1985.

[11] Henri Gillet and Christophe Soulé, An arithmetic Riemann-Roch theorem, Invent. Math. 110 (1992), no. 3, 473-543.

[12] H. Gillet and C. Soulé, Analytic torsion and the arithmetic Todd genus, Topology 30 (1991), no. 1, 21-54. With an appendix by D. Zagier.

[13] A. Grothendieck, Éléments de géométrie algébrique. Inst. Hautes Études Sci. Publ. Math. 4, 8, 11, 17, 20, 24, 28, 32 (1960-1967).

[14] Robin Hartshorne, Algebraic geometry, Springer-Verlag, New York, 1977. Graduate Texts in Mathematics, No. 52.

[15] Daniel Huybrechts, Fourier-Mukai transforms in algebraic geometry, Oxford Mathematical Monographs, The Clarendon Press Oxford University Press, Oxford, 2006.

[16] An-Min Li and Yongbin Ruan, Symplectic surgery and Gromov-Witten invariants of Calabi-Yau 3-folds, Invent. Math. 145 (2001), no. 1, 151-218.

[17] Eduard Looijenga, Motivic measures, Astérisque 276 (2002), 267-297. Séminaire Bourbaki, Vol. 1999/2000.

[18] Vincent Maillot and Damian Roessler, On the periods of motives with complex multiplication and a conjecture of Gross-Deligne, Ann. of Math. (2) 160 (2004), no. 2, 727-754. 
[19] Ju. I. Manin, Correspondences, motifs and monoidal transformations, Mat. Sb. (N.S.) 77 (119) (1968), 475-507 (Russian).

[20] D. O. Orlov, Derived categories of coherent sheaves and equivalences between them, Uspekhi Mat. Nauk 58 (2003), no. 3(351), 89-172 (Russian, with Russian summary); English transl., Russian Math. Surveys 58 (2003), no. 3, 511-591.

[21] Christophe Soulé, Genres de Todd et valeurs aux entiers des dérivées de fonctions L, Astérisque 311 (2007), Exp. No. 955, vii, 75-98 (French, with French summary). Séminaire Bourbaki. Vol. 2005/2006.

[22] Lucien Szpiro (ed.), Séminaire sur les pinceaux arithmétiques: la conjecture de Mordell, Société Mathématique de France, Paris, 1985. Papers from the seminar held at the École Normale Supérieure, Paris, 1983-84; Astérisque No. 127 (1985).

[23] Jarosław Włodarczyk, Toroidal varieties and the weak factorization theorem, Invent. Math. 154 (2003), no. 2, 223-331.

[24] Ken-Ichi Yoshikawa, Analytic torsion and an invariant of Calabi-Yau threefold, Differential geometry and physics, Nankai Tracts Math., vol. 10, World Sci. Publ., Hackensack, NJ, 2006, pp. 480-489. 